\title{
Introduction to Inter-duality Theory
}

\section{----An underlying way to reveal the nature of general system}

\author{
Shaokun Chen \\ Institute for Ultimately Objective World and Systematics Research, Chengdu, China \\ chenshaokun1989@gmail.com
}

\begin{abstract}
This paper intends to reveal the nature of a system concept and the intrinsic structure of a general system. Furthermore, the inter-duality theory will be introduced concisely and employed to analyze the nature, behavior and principle of a management system.
\end{abstract}

Index Terms - general system, nature, structure, inter-duality

\section{I . Introduction}

The birth of Systems Science has had epoch-making significance, which, in terms of philosophy, indicates that the human scientific process has converted from the mechanical reductionism era, with the feature of "1+1=2", into a new age, the systematical thinking era, holding the characteristic of $" 1+1 \neq 2 "$.

Meanwhile, a new epoch of Human Sciences has been switched into Post-positivism, i.e. a theory can be established on the basis of a set of conjectures (or axioms) instead of finding entirely available evidence (just accepting the logical verification and striving to gain wholly solid evidence). Seriously, almost all modern theories have these postpositivism characteristics, such as mathematics, microphysics, cosmology, social sciences, and even include theology and philosophy etc. For instance, Newtonian mechanics was built on three postulates; Einstein's Theory of Relativity was initially constructed by two axioms, which had not been verified at that time; including the postulates of economics and laws and the regulations and rules in management activities.

We integrate the axiomatization and formalization into systematical insights and axiomatic thought, which is naturally employed as a new thinking style for cognizing and modeling the objective objects---systems. This axiomatic thought should absolutely be introduced into management science, social science, theology, philosophy and systems science, etc.

Human Sciences have evolved for thousands of years, and we have created a range of thoughts, methods and models to make sense of the laws and principles of physical reality, the behavior of living systems, the activities of humanity and the phenomena of abstract existence, etc. Actually, from the viewpoint of General System Theory [1], extracted from sophisticated phenomena and complex objects and intricate human activities and proposed explicitly by Ludwig Von Bertalanffy, it is evidently reasonable that existence or reality or entity, in terms of generalized category, can be accurately described as a system. Therefore, it is unquestionable that we can employ revealing the nature and intrinsic structure and evolution of a general system to represent the study of essence, laws and principles of objects involved in an Ultimately Objective World [2].

Roughly speaking, in Europe, the material systems are main research field, and in America, they concentrate on living systems, while in China, we focus mainly on social systems. In summary, the theoretical structure of systems science basically involves four fields: the systematic philosophy, the systematic theories, the systematic engineering and the systematics, as shown in Figure 1.

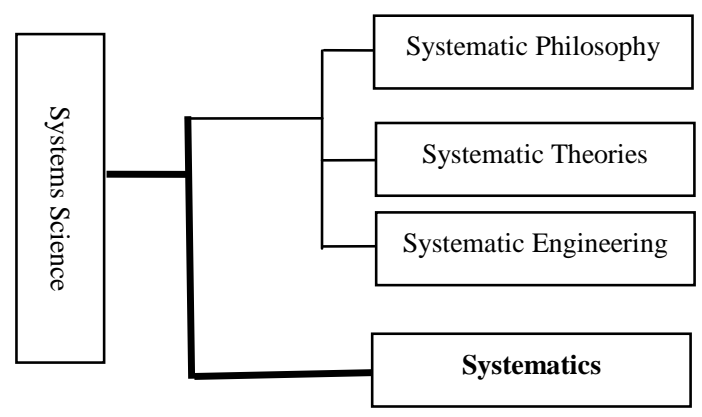

Fig. 1 the structure of Systems Science

In this paper, we concentrate on an essential theory of systematics, which is the fundamental theory of systems science [3]. There are three parts to our research of the nature, the intrinsic structure of the general system and the application study based on a management system of inter-duality theory:

1) Concept Study: Introduce definitions and descriptions of the concept of a general system.

2) Structure Study: Reveal the nature or the intrinsic structure of an arbitrary system and propose the inter-duality concept and introduce the inter-duality theory.

3) Application Study: Apply inter-duality thought to reveal the nature and principle of a management system.

\section{II . Concept Study}

Any independent science has its fundamental theory [3],[4].

\section{A . Concept of Systematics}

Systematics or systematology, like mathematics, physics, sociology, politics etc., is a fundamental theory corresponding to an independent science. There are three common features:

1) To explore the general problems;

2) To explain the conjunct phenomena; 
3) To reveal and establish a fundamental logic system and to have higher abstraction and more profundity.

Ultimately, a fundamental theory must deal with basic concepts and answer the question of "being" or reveal the nature of associated objects instead of modeling or merely defining and explaining these objective objects. This is the distinct difference between reality and approximation or essential otherness between conception and definition, shown as follows:

1) Conception is the real reflection of objective world through consciousness behavior; its characteristics are "objectivity", "uniqueness" and "being", i.e., one cognizes the system from answering "what it is actually being".

2) Definition is the description of a concept by concise words in order to facilitate communication; its features are "subjectivity", "non-uniqueness", "conciseness", and approximation i.e., one uses definition to approximate a concept as close as possible and aims at answering "what it is really like".

In general, the definition is divided into "descriptive definition"and "axiomatic definition" [5].

Accordingly, a definition of systematics is provided.

Definition 1: Systematics is a basic theoretical branch of systems science, which satisfies the following axioms:

1) In allusion to the most widely objective objects involved in systems science, i.e., the general systems;

2) The research objects are a "complete system" which is based on Ultimately Objective World View [2][3][5].

3) The research objectives are revealing the nature of the system and its intrinsic structure, the fundamental laws and principles of the general system, i.e., exploring the being cognition and complete space investigation of systems.

4) To create and to apply the most profound and powerful methodology, inter-duality theory.

\section{$B$. Concept of general System}

As the concept of "system" has been proposed for almost 80 years, there must be many definitions from different fields with various descriptions [6]. Therefore, in this paper, it is reasonable that we can define a "system" as follows:

Definition 2: An object is a system if it is treated as something that contains contents and details.

By explanation, we usually say "a system consists of elements". Through the above definition, we know that this sentence in quotation marks is not a system, while it actually is a system, once one focuses on its contents and details. It is true that from the viewpoint of nowadays a system is made up of elements, yet we concentrate more on this "constitution" with respect to the system. That is, we focus on firstly, what is the result of this constitution; and secondly, what is the constitution itself. It is reasonable from definitions as follows that the "constitution" means forming a system through relationship. Therefore, it is of more significance to explore the relationships of a system.
Definition 3: A system is a triple group.

This definition means that a system is a triple structure that consists of its "objective space", "elements space" and "relationship space".

Let a system be $S$, then it can be defined as a set form:

$$
S=(Y ; X, F)
$$

Here $Y$ represents the objective space of a system; $X$ expresses the elements space of a system; $Y$ indicates the relationship space of a system. Further, we understand:

Definition 4: A system can be equally represented by its objective spaces.

Let a system be $S$, then we get,

$$
S=(Y ; X, F)=\{y: y=f(x, a), y \in Y, x \in X,(f, a) \in F\}
$$

Therefore, a system can be denoted as $S=Y=\{y\}$.

Proposition 1: A system can be embodied by subsystems of different hierarchies.

Let a system be $S$, and its subsystems are $\{s\}$, then for $S$, it has its own objective space and we can denote it as:

$$
s=y=f(x, a), y \in Y ; x \in X ;(f, a) \in F
$$

Therefore, $S=\{s\}=\{y\}$.

In principle, an arbitrary system $S$ can be an element of its higher hierarchical system; meanwhile, it also occupies its lower hierarchical systems as its elements. So is the view of the objective spaces of a system.

Definition 5: An objective system is an open system.

Generally speaking, a system exists in its environment or takes a higher level or deeper hierarchical system as its circumstance; furthermore, a system has the capacity to exchange energy and information and substances with its environment. Therefore, it is evidently reasonable that an objective system must be an open system.

In this part, the definitions of the concept of a general system have been constructed. The next parts will reveal the nature of a general system and do a profound study on the intrinsic structure of a system.

\section{Structure Study}

Although we have established the definitions of the concept of a general system, we have not yet revealed the nature and intrinsic structure and inner properties of systems. This section will explain these definitions.

\section{A. Inter-dual System}

Under the description of segment 2, we have to ask further: whether the notion of a system could excavate deeper and more profound? It can be realized that we would explore and reveal a system from its abstract and virtual hierarchies. Indeed, we can analyze a system from its spatial hierarchies. Meanwhile, if we use spatial consciousness to rethink the 
characteristics of profoundly investigating systems, it is obvious that the methods of analysis of a system are usually manifested as a "flat thinking style", such as row and column, item and piece, far and near, inside and outside, domain and range, scope and region, etc. Sometimes these styles mention the hierarchy, gradation, vertical, jump, etc., but it is merely through sub-consciousness, e.g., from the meaning of daily life and the sense of experienced thinking.

Therefore, we need the breakthrough of spatial consciousness, especially when studying the complete space of a system and inter-duality of a general system.

From definition 3 and 4, proposition 1, we can easily demonstrate that if a system is denoted by $S$, and all its subspaces the system occupies are denoted by $\left\{s_{i j}\right\}$, we get:

$$
S=\left\{S_{i j}\right\}=\left\{S_{i j}\right\}_{i, j=1}^{m, n}
$$

Here, $i$ denotes spatial hierarchies of subsystems and $j$ denotes the number of subsystems in the same level.

Proposition 2: A system encompasses completely spatial hierarchies of the system or complete space of the system.

From (4) and proposition 1, we can verify succinctly that the completely spatial hierarchies determined by all the subsystems of a general system integrate into complete space of the objective system. In this way, we understand clearly and demonstrate vividly that the "complete space" of a general system can be an underlying notion to recognize the general system visually and analyze the objective system profoundly.

Moreover, for $\forall s_{i_{1}}, s_{i_{2}}$, if $s_{i_{2}}$ is a higher hierarchical system related to $S_{i_{1}}$, we can get $: S_{i_{1}} \neq S_{i_{2}}$, and $S_{i_{2}}$ has a higher spatial level than $S_{i_{1}}$. In this case, we denoted $S_{i_{2}}$ as the abstraction or functional or promotion of $S_{i_{1}}$. That is: for $S_{i_{1}}$ and $S_{i_{2}}$, there is a mapping:

$$
\wp_{i}: S_{i_{1}} \rightarrow S_{i_{2}}
$$

Therefore, considering the different hierarchical subsystems of a system, there are distinct relationships between them in terms of this system. In depth, if a subsystem $S_{2}$ is the abstraction or functional or a higher spatial hierarchy with respect to a subsystem $S_{1}$ of a system, we denote $S_{1}$ as real-like ( or real space), while $S_{2}$ is imaginary-like (or imaginary space); furthermore, we denote the "real-like" and "imaginary-like" duality as inter-duality. In this sense, the real spaces and imaginary spaces involving in a general system integrated into a whole can be a complete space of the system.

Accordingly, the terms "real-like" and "imaginary-like" are relative concepts and we can distinguish them from conceptual or ideational meaning. Furthermore, the real-like and imaginary-like concepts in a system are non-uniqueness, for instance, the pair of an entity and its attributes or the elements and relationship duality of this entity, etc.

Definition 6: A system is an inter-dual system if it comprises inseparable real-like and imaginary-like duality that is inextricably intertwined.

Let a system be an inter-dual system, denoted as $S$, and let real-like be $X$, while imaginary-like is $X^{*}$, then:

$$
S=\left(X^{*}, X\right)
$$

The inseparable duality of real-like and imaginary-like in a system, such as a concrete image and an abstract image of a system, a real image and an imaginary image of a system, an actual image and a virtual image of a system, or a hard part and a soft part of a system etc., can be denoted as inter-duality.

As is shown above, we introduce a mapping $\wp$ to present the connection and distinction between real-like $X$ (real space) and imaginary-like $X^{*}$ (imaginary space) with respect to a system $S$. That is:

$$
\wp: X \rightarrow X^{*} \text { or } \wp_{i}: X \rightarrow x_{i}^{*}, x_{i}^{*} \in X^{*}
$$

It is obvious that the real-like and imaginary-like duality must be essentially different, i.e., an element of imaginary space, at least, is the abstraction, functional and global mapping with respect to the real space, i.e., $x^{*}=\wp(\mathrm{X})$.

Proposition 3: An inter-dual system is a complete system.

From proposition 2, we know that a system contains its entirely spatial hierarchies that can be denoted as real-space and imaginary-space, which are integrated as a complete space of the system. It can be expressed as $S=\left(X^{*}, X\right)=\left\{s_{i j}\right\}$, i.e., an arbitrary system must be a complete system with an intrinsic structure of inter-duality. Actually, inter-duality is just one intrinsic structure of general system, but it is at least, from my point of view, an underlying way to reveal the nature of a general system and, furthermore, it is the intrinsic mechanism to generate or to create the inner power or inherent impetus of the configuration or formation of a system. Thirdly, inter-duality is the fundamental structure that drives a system evolution and development or destruction.

Proposition 4: Prove $(Y ; X, F) \Leftrightarrow\left(X^{*}, X\right)$, for a system $S$.

Prove: for a system $S$, the elements and real-like are the same, i.e., $X=X ; \because S=Y, Y=\{y\}$, for $\forall y$, it satisfies $\{y\}=\{y: y=f(x, a), y \in Y, x \in X,(f, a) \in F\}$; for $X$ and $X^{*}$, there exists $\wp: X \rightarrow X^{*} ; \therefore X^{*}=(Y ; F)$. Therefore: $(Y ; X, F) \Leftrightarrow\left(X^{*}, X\right)$.

This proposition is also a demonstration that the objective space and relationship space of an arbitrary system must stand at the imaginary-like or imaginary space of the system. From this viewpoint, it is powerful and profound that the inner nature and global behavior of objective space and relationship 
space of general systems are revealed based on inter-duality thought and the breakthrough of spatial consciousness.

\section{B. Inter-duality Theory}

As we have introduced and constructed the concept of an inter-dual system, it is reasonable that the properties and principles should be established in an underlying and general way. It is better to present these ideas using theorems:

Theorem 1: An arbitrary system inherently has an interdual structure, and the real-like and the imaginary-like elements of the system must be concurrently emerging and simultaneously vanishing - that is to say, they are inextricably intertwined and cannot be divorced from each other.

It can be easily proven using definition 6. Essentially, the relationship between real-like and imaginary-like must be nonlinear and none of them can be zero, i.e., inter-duality is the underlying structure and essential law of a general system.

Theorem 2: The real-like and the imaginary-like elements of an inter-dual system must exist spatial hierarchical difference.

According to definition 3 and 4 and from proposition 4, $S=(Y ; X, F) \Leftrightarrow\left(X^{*}, X\right)$, it is obvious that the objective space and relationship space of a general system must be substantial and intrinsic difference with respect to its elements space. Therefore, it is true that the imaginary-like $X^{*}$ and the real-like $X$ of an inter-dual system are essentially spatial hierarchical difference.

Moreover, from mapping $\wp: X \rightarrow X^{*}$, we denote $X^{*}$ as the dual space with respect to $X$. Mathematically, in terms of linear functional, a dual space is defined as follows:

Let $X$ be a linear space, if there is another space $X^{*}$, and they satisfy the inner product (denoted as $\langle\bullet, \bullet\rangle$ ), i.e., for $x_{1}, x_{2} \in X ; x_{1}^{*}, x_{2}^{*} \in X^{*} ; a, b \in R$, then

1) $\left\langle x_{1}^{*}, a x_{1}+b x_{2}\right\rangle=a\left\langle x_{1}^{*}, x_{1}\right\rangle+b\left\langle x_{1}^{*}, x_{2}\right\rangle$,

$$
\text { or }\left\langle a x_{1}^{*}+b x_{2}^{*}, x_{1}\right\rangle=a\left\langle x_{1}^{*}, x_{1}\right\rangle+b\left\langle x_{2}^{*}, x_{1}\right\rangle \text {; }
$$

2) for $x_{0} \in X$, if for $\forall x^{*} \in X^{*}$, there is $\left\langle x_{0}, x^{*}\right\rangle=0$, then $x_{0}=0$, or for $x_{0}{ }^{*} \in X^{*}$, if for $\forall x \in X$, there is $\left\langle x_{0}{ }^{*}, x\right\rangle=0$, then $x_{0}{ }^{*}=0$;

And here, $X^{*}$ is the dual space of $X$, denoted as $\left(X^{*}, X\right)$.

Therefore, the inter-duality, also denoted as $\left(X^{*}, X\right)$, of a general system is generalized from linear functional, and from the definition of the concept of dual space, $X^{*}$ is the set of all functional, denoted as $\wp=\left\{\wp_{i}\right\}$, and $\wp_{i}: X \rightarrow R$, then, $\wp(X, R) \stackrel{\Delta}{=} X^{*}$. Obviously, the imaginary-like $X^{*}$ and the real-like $X$ must exist spatial hierarchical difference.

Theorem 3: The real-like and imaginary-like elements of an inter-dual system must possess an intrinsic duality relationship or inter-duality relationship.

Let real-like be $X$ and imaginary-like be $X^{*}$, due to definition 6 and the description of theorem 2, the real space is $X$, then imaginary space $X^{*}$ can be:

$$
X^{*}=\wp(X) \text { or } X^{*}=\left\{X_{i}^{*}\right\}=\wp_{i}(X)
$$

It is reasonable that $\wp$ can be an operator between realspace $X$ and imaginary-space $X^{*}$, i.e.:

$$
\wp_{i}(X)=x_{i}^{*}, x_{i}^{*} \in X^{*}
$$

According to (9), we can also know the difference between the real-like and imaginary-like, and we can unquestionably define the difference as opposite action in an inter-dual system. Meanwhile, an inter-dual system must be unified underlying this inextricably and inseparably opposite operation, therefore, we denote this unification behavior as unified action according to the general system. In conclusion, the simultaneous opposite and unified action can be denoted as inter-duality relationship, as shown in Figure 2:

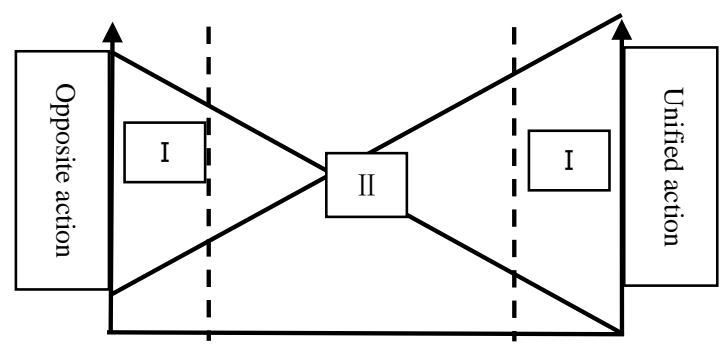

Fig. 2 the inter-duality relationship

The opposite action and unified action relationship of inter-duality are intrinsic duality relationship and inextricably interaction. This inter-duality relationship of an arbitrary system is concurrently emerging and simultaneously vanishing as is consistent with the real-like and imaginary-like of an inter-dual system.

Corollary 1: Opposite action generates dynamic or impetus, while unified action creates progress or advance.

Because of the intrinsic difference of an inter-dual system and this essential difference is the potential of the system, the real-like and imaginary-like can inherently evolve. This is the reason of why a system moving and evolving. Of course, due to this impetus, a system can reach and achieve its systematic equilibrium, and this is the progress or advance that results from the unified action.

Theorem 4: The real-like and imaginary-like elements of an inter-dual system must have an inherently interactive relationship or interaction of inter-duality. 
Let $X=\{x\}, X^{*}=\left\{x_{i}^{*}\right\}=\wp_{i}(X)$, if there is any change of real-like $X$ or imaginary-like $X^{*}$, the other must be inherently changing to satisfy its inner equilibrium state.

We can also present the change as $\hat{X}=X+\Delta_{i}$, then $\wp(\hat{X})=x^{*}+\Delta_{i}^{*}$, that is, $X^{*}$ can also be changed $\Delta_{i}^{*}$ in this action, vice versa. This reciprocally intrinsic change can be defined as interactive relation of an inter-dual system, or denoted as interaction of inter-duality.

Theorem 5: The real-like and imaginary-like elements of an inter-dual system must exist in a proportional relationship or flexibility of inter-duality.

Let real-space be $X$, and imaginary-space be $X^{*}$; there must exist a mapping $F_{i}: X \rightarrow R ; F_{j}: X^{*} \rightarrow R$, then $F_{i}: F_{j}=\alpha \in(\delta-\varepsilon, \delta+\varepsilon)$, and $(\delta-\varepsilon, \delta+\varepsilon)$ is denoted as the domain or interval of flexibility of inter-duality.

Corollary 2: The inter-dual structure of a general system is multilayered structure of fractal modality.

In general, the real-like and imaginary-like elements themselves can be an inter-dual system. Philosophically, the inter-dual structure of any general system could consist of infinite hierarchies. Therefore, a system could be the same structural formation in a fractal pathway or iteration way.

Corollary 3: A general system must be a complex system.

In principle, as arbitrary system can be consisting of different hierarchies of subsystems and these subsystems can also be regarded as inter-dual structure. Moreover, the relationship between subsystems, generally speaking, is nonlinear or irreversible or uncertain or combination of these properties. Therefore, a general system should be treated as a complex system. In other words, the inter-duality theory is a theory of complex systems.

Inter-duality Theory: when a system is investigated using inter-duality thought, it is inter-dual system, and the theory of revealing the inter-dual structure and inter-dual behavior and inter-dual mechanism etc., is denoted as interduality theory.

The inter-duality theory, roughly speaking, would be applied to many fields, such as mathematics, physics, politics, sociology, management science and systematics etc.

For instance, from the theory of inter-duality, we can reveal the nature of a mathematical model, especially the parameter space in various models[2],[3],[5],[7] reveal the nature of space-time structure, the origin and essence of logic, the essence of humanity, the essential structure of particles, the nature of consciousness etc.,[2],[3],[5],[7],[8],[9]. Further, we have investigated the nature of society, the principles and essence of generalized measurement, the nature and principles of management etc.,[2][4][10].

\section{IV . Application Study}

As an application, let's consider the representative inter- dual system, the management system, and we will reveal its nature and operational law.

In order to study the nature and law of management systems, it is effectively to denote a management system as manager system $M^{*}$ and managed system $M$.

For this analysis, we merely concentrate on the standard management process, a management system that holds its exact objective space $O$, managed space $M$ and the relationship space $F$ between $O$ and $M$; meanwhile it involves one periodicity of management action. From the view of the inter-duality theory, the $O$ and $F$ are imaginary-like $M^{*}$ (manager system), while the $M$ is real-like (managed system), in terms of an inter-dual management system $S_{M}$.

Then, we denote the standard management process is the mapping process: $M_{i} \stackrel{\wp_{i}}{\rightarrow} m_{i}^{*} \stackrel{\wp_{i}^{*}}{\rightarrow} M_{j}$, i.e., the first operation $\wp$ is management with the drive from the managed system $M$, while the second management $\wp^{*}$ is motivated by the manager system $M^{*}$. We define this continuous management process as the interaction of inter-duality.

Proposition 5: If a management system is in its optimization state, or in the inter-dual equilibrium state, there is no need to run a management behavior.

Because there is no change in imaginary-like or real-like according to our hypothesis. By contrast, if there is any change in this inter-dual system, there must be a management behavior to adjust and adapt the system to a new equilibrium or new optimization.

Theorem 6: The nature of management is to achieve the optimization of the real-like from the operation of the imaginary-like in a management system.

Corollary 4: The management behavior is to maintain the equilibrium by the means of the interaction of inter-duality in the management system.

Therefore, a management system can be denoted as its objective space or its imaginary-like, such as, management is decision, or management is control etc. It is without doubt that a management system is

$$
S_{M}=\left(M^{*}, M\right)=(O ; M, F)
$$

Operator $\wp$ represents the first management:

$$
\wp_{i}: M_{i} \rightarrow m_{i}^{*}, m_{i}^{*} \in M^{*}
$$

Where $\wp$ denotes an operation process based on $M_{i}$. The inverse operator $\wp^{*}$, as the second management, satisfies:

$$
\wp_{j}^{*}: m_{i}^{*} \rightarrow M_{j}
$$

Further, we can conclude that, 


$$
\left\{M_{i j}\right\}=\left\{\wp_{j}^{*}\left(\wp_{i}\left(M_{i}\right)\right)\right\}=\wp^{*} \circ \wp \circ M
$$

According to the description of a standard management process, denoted as $M_{i j}$, it is clearly indicated that a management behavior is always a series of $M_{i j}$. Further, $M_{i j}$ is an inter-dual dynamical system, due to the interaction of inter-duality.

For the dynamical process of an inter-dual management system, as in a management mathematical application, it is useful that an inter-dual dynamical system of management can be established at this proper position.

The current management system can be presented as:

$$
O_{i}=F_{i}\left(M_{i}, \varepsilon_{i}\right)
$$

Where, $\varepsilon_{i}$ is the change of imaginary-like with respect to the operation of $\wp$, i.e., the manager system makes a valuation and decision on the first process of management, which is driven by the inner difference of managed system relative to its objective space or the optimization state. When it comes to the action from a manager system to a managed system, there should be an inter-dual intrinsic change $\varepsilon_{j}{ }^{*}$ of real-like result from the change of imaginary-like $\varepsilon_{i}$ and the operation $\wp^{*}$. Essentially, $\varepsilon_{i}$ and $\varepsilon_{j}{ }^{*}$ are merely the fine adjustments underlying the same strategic structure, i.e., the macrostructure is not changed in this management dynamical process. Therefore, we get:

$$
O_{i}=F_{i}\left(M_{j}, \varepsilon_{j}^{*}\right)
$$

According to the description, we can naturally reveal the interaction of the inter-dual fluctuation, i.e., an inherent impetus determined by an inter-dual management system drives any perturbation into an equilibrium state, shown briefly in Figure 3.

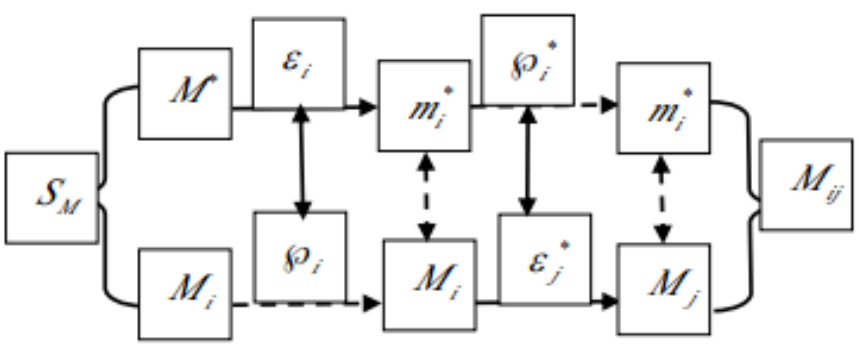

Fig. 3 the brief illustration of interaction of inter-duality

This dynamic behavior of an inter-dual system is also defined as self-organization. Therefore, we reveal another definition of the nature of management:

Definition 7: Management is a specific self-organization process.
As we can see, there are many essential and powerful laws and conclusions with the aid of inter-duality theory. Furthermore, there are many mathematical analysis methods which reflect these management natural processes, such as the Fiber Bundles[11], the Functional Theories[12], Dynamical System Theories[13], Inter-dual analysis methods[4], [10], etc.

Due to the aims and the limitations of this paper, other essential problems and fundamental principles, such as the paradox of management, the spatial relationship between the manager system and the managed system, etc., cannot be completely studied. We will reveal them and analyze them in another convenient place.

\section{Conclusion}

This paper concentrates solely on the conceptual study of revealing the nature and intrinsic structure of general systems from the viewpoint of an inter-duality theory. Therefore, there is limited introduction relative to the laws, principles and methods of objective systems, for instance, the rigorous proof of each theorem, corollary, lemma and propositions. Also, there is less study of the relationship between two systems compound inter-dual systems, or multilayered systems, etc. Moreover, we do not study the laws of evolution and development or destruction in an inter-dual system, such as social systems, physical systems, mathematical systems and the environment or neighborhood investigations, etc.

Additionally, in this paper we study the inter-dual system as a bold attempt in a manner that leads us to additional structures and further methodology on system theories. Essentially, we initiated and proposed the inter-duality theory in order that we should do further study this ultimately objective world in a Being way and using inter-duality thought, not merely the Modeling methods.

Although. The Inter-duality Theory was proposed originally at the end of the 20th century by professor Longchang Gao, it has not been popularized and has not been widely received.

Therefore, we want to present a brief illustration of the development of this theory, as shown here:

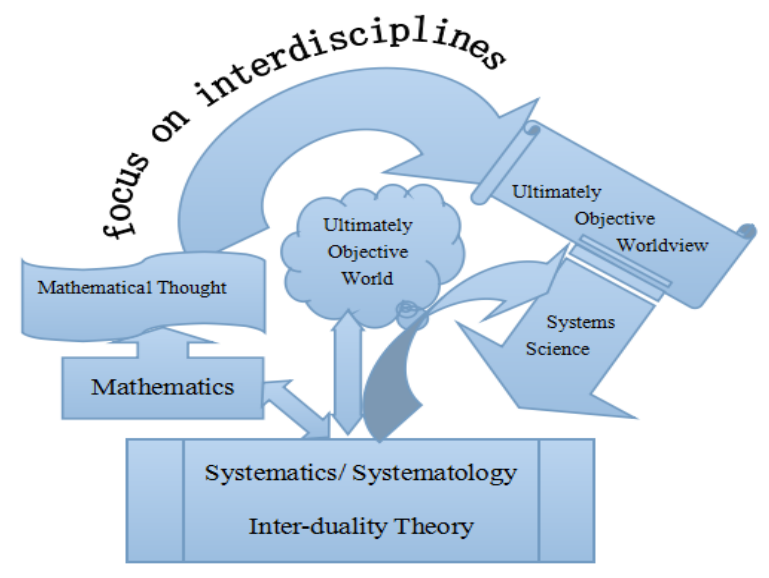

Fig. 4 brief illustration of inter-duality theory 


\section{Acknowledgment}

This paper is supported by the Institute for Ultimately Objective World and Systematics Research. The author is gratefully indebted to Professor Longchang Gao and grateful for his careful guidance and detailed suggestions.

\section{References}

[1] Ludwig Von Bertalanffy, "General System Theory------foundations, development, applications”, George Braziller, Inc. New York, 1973.

[2] Longchang Gao, "Complexity Principles in Ultimately Objective World", Science Press, 2004.

[3] Longchang Gao, "the Principles of Systematics", $2^{\text {nd }}$ ed, Science Press, 2010, pp. 2-16.

[4] Fei Xu, Longchang Gao, "Interdual Space and Management Inter-duality Theory------an fundamental exploration of management sciences”, Science Press, 2005.
[5] Longchang Gao, Wei Li, "Mathematics and Its Cognition", $2^{\text {nd }}$ ed, SWJTU Press, 2011.

[6] Changgen Piao, “Introduction to Systematology”, Shanghai Ci-shu Press, 2005, pp. 74 .

[7] Longchang Gao, Yuan Yang, "Fundamental Theory of Mathematical Modeling”, Science Press, 2007.

[8] Longchang Gao, Ran Tao,"The Competition Principles of Market Economy”, Railway of China Press, 2002.

[9] Longchang Gao etc., "Introduction of Cognition Science", SWJTU Press, 2004.

[10] Longchang Gao, "Principles of Social Measurement”, SWJTU Press, 2000.

[11] Dale Husemoller, “Fiber Bundles”, $3^{\text {rd }}$ ed, Springer Press, 1993.

[12] Ruth F. Curtain, A.J. Pritchard, "Functional Analysis in Modern Applied Mathematics", Academic Press, 1977.

[13] Jurgen Jost, "Dynamical Systems---Examples of Complex Behaviour", Springer Press, 2005. 